\title{
Vigilancia intensificada de enfermedades durante los incendios forestales, Paraguay, 2019
}

\author{
Sandra Irala ${ }^{1}$, Mónica Arellano $(\mathbb{D})$, Fátima Vázquez ${ }^{1,2}$, Katia Peralta ${ }^{1}$, \\ María Esther Pedrozo iD 1 \\ ${ }^{1}$ Ministerio de Salud Pública y Bienestar Social, Dirección General de Vigilancia de la Salud, \\ Centro Nacional de Información Epidemiológica. Asunción, Paraguay \\ ${ }^{2}$ Universidad Nacional de Asunción. Facultad de Enfermería y Obstetricia. San Lorenzo, Paraguay \\ Cómo referenciar este artículo/ \\ How to reference this article: \\ Irala S, Arellano M, Vázquez F, Peralta K, Pedrozo ME. \\ Vigilancia intensificada de enfermedades durante los \\ incendios forestales Paraguay, 2019. Mem. Inst. \\ Investig. Cienc. Salud. 2021; 19(3): 25-34
}

\section{RES U ME N}

El humo de los incendios forestales puede influir en la mortalidad, las emergencias y las visitas ambulatorias, especialmente en personas con problemas respiratorios y cardiovasculares. La vigilancia intensificada exige sumar recursos adicionales a las actividades rutinarias de vigilancia. En agosto del 2019, se registraron 25.299 focos de incendios en varios departamentos del Paraguay. Se describen los hallazgos de la vigilancia intensificada de enfermedades en la población afectada por incendios forestales en Paraguay en el 2019. Se realizó un estudio descriptivo trasversal entre el 15/08/19 al 4/10/2019 en todo el territorio nacional. Como fuente de datos, se incluyen reportes de las Unidades Epidemiológicas Regionales, organizados en una base de datos con las variables: departamento, distrito, población indígena, número de atenciones médicas, motivo de consulta, heridos graves, desaparecidos y fallecidos, identificación de riesgos potenciales para la salud. Fueron afectados 12 (12/17) departamentos y la Capital del país, 30 distritos y 12 comunidades indígenas. Se realizaron 1.681 atenciones médicas, de los cuales 1.291 (77\%) eran de Alto Paraguay. Las enfermedades más frecuentes fueron Infecciones Respiratorias Agudas no neumonías (36\%), y Enfermedades Tipo Influenza (18\%). No se registraron heridos graves, personas desaparecidas ni fallecidas. Como riesgos potenciales para la salud: pérdidas de viviendas en comunidades indígenas, cultivos, ganados y la interrupción de provisión de agua. En conclusión, los cuadros respiratorios fueron más frecuentes durante los incendios forestales, no se registraron personas desaparecidas ni fallecidas. Se identificaron riesgos potenciales para la salud por afectación de medios de vida y sustento, sobre todo en poblaciones indígenas.

Palabras clave: desastres ambientales, incendios forestales, vigilancia intensificada, Paraguay.

\section{Intensified disease surveillance during forest fires Paraguay, 2019}

\footnotetext{
A B S T R A C T

Smoke from wildfires can influence mortality, emergencies and outpatient visits, especially in people with respiratory and cardiovascular problems. Intensified surveillance requires adding up additional resources to routine surveillance activities. In August 2019, 25,299 fire outbreaks were registered in de various departments Fecha de recepción: agosto 2021. Fecha de aceptación: octubre 2021

*Autor correspondiente: María Esther Pedrozo Torres. Ministerio de Salud Pública y Bienestar Social, Dirección General de Vigilancia de la Salud, Centro Nacional de Información Epidemiológica. Asunción, Paraguay

Email: esther.pedrozo@mspbs.gov.py
} 
Paraguay. The findings of the intensified surveillance of diseases in the population affected by wildfires in Paraguay in 2019 are described. A descriptive cross-sectional study was carried out between $08 / 15 / 19$ to $10 / 4 / 2019$ throughout the national territory. As a data source, reports from the Regional Epidemiological Units, organized in a database with the variables: department, district, indigenous populations, number of medical care, reason for consultation, seriously injured, missing and deceased people, identification of potential health risks. Twelve (12/17) departments and the capital of the country, 30 districts and 12 indigenous peoples were affected. One thousand six hundred and eighty-one medical services were performed, of which $1,291(77 \%)$ were from Alto Paraguay. The most frequent diseases were nonpneumonia Acute Respiratory Infections (36\%), and Influenza-like illness (18\%). There were no injured, missing or dead people. Among the potential health risks: loss of dwellings in indigenous people, crops, livestock and the interruption of water supply. In conclusion, respiratory symptoms were the most frequent during the wildfires, there were no injured, missing or dead people. Potential health risks were identified due to the impact on livelihoods, especially in indigenous populations.

Keywords: environmental disasters, wildfires, intensified surveillance, Paraguay

\section{INTRODUCCIÓN}

Los incendios forestales son una parte importante del ecosistema forestal. Pero a causa de los cambios climáticos y del uso (y abuso) humano del fuego, los incendios son ahora una amenaza para muchos bosques y su biodiversidad ${ }^{(1)}$. El humo corresponde a una modalidad de exposición aguda a la contaminación atmosférica, cuya aparición y evolución es difícil de $\operatorname{prever}^{(1,2)}$. Las principales emisiones primarias de los incendios forestales que empeoran la calidad del aire incluyen partículas del aire ambiental como partículas finas y gruesas (PM) y gases, monóxido de carbono (CO), metano, óxido nitroso $\left(\mathrm{N}_{2} \mathrm{O}\right)$, óxidos de nitrógeno (NOx), carbono orgánico volátil (COV), así como muchos otros tóxicos del aire ${ }^{(3)}$.

Diversos estudios, concluyen que existe una asociación positiva entre la exposición al humo de incendios forestales y mortalidad por todas las causas y morbilidad respiratoria ${ }^{(4,5)}$. La morbilidad respiratoria incluye asma, enfermedad pulmonar obstructiva crónica (EPOC), bronquitis y neumonía ${ }^{(4)}$. Igualmente, de ser un factor de riesgo de efectos cardiovasculares adversos, especialmente entre las poblaciones susceptibles, incluidos los ancianos, las mujeres embarazadas y las personas con bajo nivel socioeconómico ${ }^{(6)}$.

Se estima que la mortalidad media atribuible a la exposición al humo de incendios forestales a nivel global es 339.000 (RIC: 266.000 a 600.000) muertes anuales. Siendo las regiones más afectadas, África subsahariana (157.000) y el sudeste asiático $(110.000)$ y en menor proporción Sudamérica $(10.000)^{(7)}$. De manera similar a otros factores de riesgo ambiental, como el agua no potable y la contaminación del aire interior y urbano, la carga de mortalidad atribuible al humo de incendios forestales recae de manera desproporcionada en las regiones de bajos ingresos del mundo ${ }^{(7)}$.

En Paraguay, los eventos relacionados al clima, y que producen efectos adversos en el ámbito social, económico y ambiental son en su mayoría los relacionados a las inundaciones, sequías, incendios forestales y tormentas severas ${ }^{(8)}$. En Paraguay, las causas de incendios forestales en su mayoría se relacionan a actividades humanas, como son el cambio del uso de tierra, desmonte, mantenimiento de pastizales para ganadería, entre otros ${ }^{(8)}$. En el decenio de 2007-2017, se identificaron en Paraguay 96 eventos por incendios forestales, en 16 departamentos ${ }^{(9)}$.

El año 2019 se caracterizó por la severidad de eventos ambientales tanto en la región de Sudamérica como en Paraguay. En Paraguay, el primer semestre estuvo marcado por intensas precipitaciones e inundaciones que obligaron al desplazamiento a poblaciones asentadas en territorios ribereños de gran parte de los departamentos. En contraste, el segundo semestre se caracterizó por una intensa sequía y persistentes incendios forestales, que afectaron principalmente al departamento de Alto Paraguay, donde se encuentran las áreas protegidas, tanto públicas como 
privadas, más grandes del país ${ }^{1}$, como en otras zonas del territorio paraguayo que fueron agravados por las condiciones climáticas adversas (altas temperaturas, vientos superiores a $20 \mathrm{~km} / \mathrm{h}$ y baja humedad) ${ }^{(10)}$.

La vigilancia intensificada, es la que se emplea en caso de brotes o ante eventos de importancia para la salud pública, que exigen sumar recursos adicionales a las actividades rutinarias de vigilancia, utiliza un tipo de búsqueda activa en establecimientos de salud y en la comunidad ${ }^{(11)}$. Vigilar las enfermedades ocasionadas por los incendios forestales es de vital importancia para evaluar el impacto en las poblaciones afectadas de modo a mitigar los efectos adversos en la salud a causa del humo producido por los incendios forestales. A continuación, se describen los hallazgos de la vigilancia intensificada de enfermedades en la población afectada por incendios forestales en Paraguay durante el año 2019.

\section{MATERIALES Y MÉTODOS}

Se realizó un estudio descriptivo de corte trasversal, entre el 15 de agosto al 4 de octubre del 2019, en todo el territorio nacional. Para identificar las zonas afectadas por los incendios forestales se realizaron verificaciones diarias de condiciones del clima, hidrología, focos de calor, proyecciones climáticas, y monitoreos diarios de prensa digital con temas relacionados al evento, con esto se identificaron las zonas afectadas para solicitar los reportes a los referentes de las Unidades Epidemiológicas Regionales (UER) de las localidades afectadas.

Como fuente de datos para la descripción epidemiológica, se utilizaron los reportes de las UER, que fueron organizados en una base de datos con variables demográficas que incluyó departamento y distrito, descripción del lugar afectado (parque nacional, establecimiento agroganadero, pastizal, otros), comunidad indígena afectada, número de atenciones médicas, motivo de consulta, desaparecidos, fallecidos, además de la identificación de riesgos potenciales para la salud.

Los departamentos fueron organizados según ejes geográficos establecidos para el monitoreo de los servicios de salud, en:

- Eje Norte (Concepción, Amambay, San Pedro, Canindeyú)

- Eje Sur (Paraguarí, Misiones, Itapuá, Ñeembucú)

- Eje Centro Este (Caazapá, Guairá, Caaguazú, Alto Paraná)

- Eje Chaco (Alto Paraguay, Boquerón, Presidente Hayes)

- Eje Metropolitano (Asunción y Central)

Para el análisis de datos, se utilizó la estadística descriptiva con números absoluto y proporciones con el programa informático Excel, y los mapas fueron elaborados con el software gvSIG.

\section{RESULTADOS}

\section{Breve cronología}

- 14 de agosto, 2019. Fueron identificados tres focos de calor dentro del Complejo de Reservas del Pantanal Paraguayo, a una distancia de $9 \mathrm{~km}$ al oeste de la Estación de Los Tres Gigantes ${ }^{2}$, y 5 focos a $15 \mathrm{~km}$ de la misma, en dirección noreste (10).

- 18 de agosto, 2019. Se reportó un incendio de gran magnitud en el en el Parque Nacional Rio Negro, Estación Tres Gigantes del departamento de Alto Paraguay. Primeros informes referían la afectación de fauna y flora locales en 61.000 hectáreas (ha) de la reserva. En la localidad de Bahía Negra distante a 40 km de la Estación Tres Gigantes, se reportó afectación de población por humo generado, siendo las afecciones principales la de vías respiratorias.

\footnotetext{
${ }^{1}$ La ecorregión del Pantanal Paraguayo se extiende a lo largo del extremo norte del país, en la margen derecha del Río Paraguay, principalmente en lo que respecta al territorio del Alto Paraguay. Se caracteriza por una vegetación de sabana herbácea, con palmares de karanda'y (Copernicia alba) como especie dominante, sujeta a inundaciones periódicas, con un régimen de precipitaciones anuales que oscila entre los 900 y 1400 mm [(Gauto et al. 2011) citado por Guyra-Paraguay] ${ }^{2}$ Reserva Pantanal Paraguayo, cuenta con 14.271 ha, donde se encuentra asentada la Estación Los Tres Gigantes, que es su centro administrativo y de visitas, es una de las áreas tituladas a nombre de Guyra Paraguay, y convertida en unidades de conservación (Guyra-Paraguay).
} 
- Se registraron incendios en departamentos del norte como Concepción, San Pedro, Amambay, Canindeyú, y del centro-este en los departamentos de Guairá y Caazapá.

- Ante estos eventos, se inició en la Dirección General de Vigilancia de la Salud, la vigilancia intensificada de enfermedades relacionada a los incendios forestales, con la finalidad de proveer información oportuna para las intervenciones en salud.

- 9 de setiembre, 2019. El Poder Ejecutivo promulgó la Ley N 6373, que declara en "Situación de Emergencia Ambiental a los departamentos de Alto Paraguay y Boquerón, de la Región Occidental" por un periodo de 60 días, tras ser afectados por los incendios forestales (12)

\section{Localidades afectadas}

El período de estudio abarco 50 días, donde se reportaron 13 departamentos incluyendo la Capital del país, con 30 distritos y 12 comunidades indígenas que fueron afectados por incendios forestales (Mapa 1 y Tabla I).

Fuente: Elaboración propia a partir de resumen de monitoreo de incendios forestales. Centro Nacional de Información Epidemiológica-DGVS.

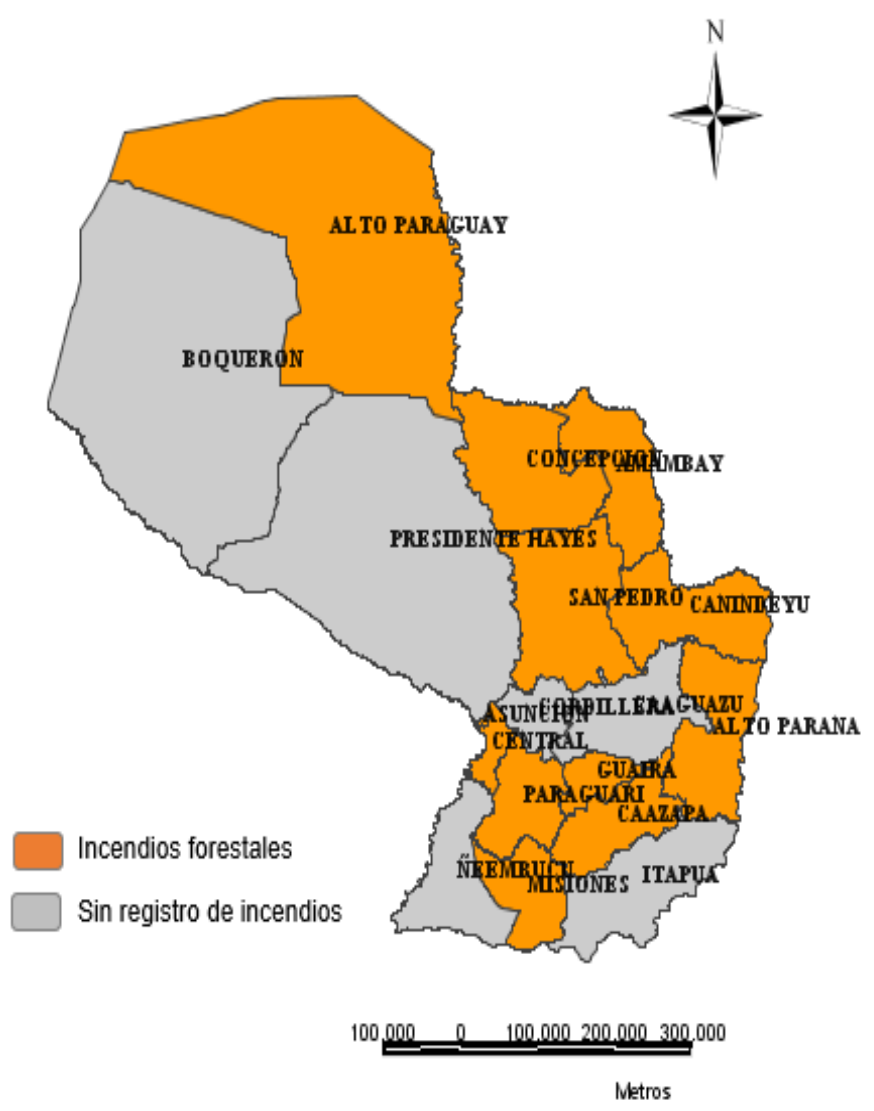

Mapa 1. Departamentos afectados por incendios forestales en Paraguay, del $15 / 08 / 2019$ al 04/10/2019. 
Tabla 1. Zonas afectadas por incendios forestales por distritos y departamentos en Paraguay, del 15/08/2019 al 04/10/2019.

\begin{tabular}{|c|c|c|c|}
\hline $\begin{array}{c}\text { Eje } \\
\text { Geog. }\end{array}$ & Departament & Distrito & Localidad \\
\hline \multirow{9}{*}{ 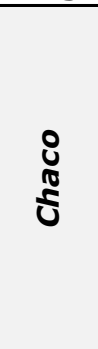 } & \multirow[t]{9}{*}{ Alto Paraguay } & \multirow[t]{5}{*}{ Bahía Negra* } & Reserva Forestal Tres Gigantes \\
\hline & & & Est. Campo Grande \\
\hline & & & Est. Uruguay \\
\hline & & & Est. Chovoreca \\
\hline & & & Cerro Chovoreca \\
\hline & & \multirow[t]{4}{*}{ Fuerte Olimpo } & Fuerte Olimpo \\
\hline & & & Toro Pampa \\
\hline & & & Est. Maranata (Ma. Auxiliadora) \\
\hline & & & Est. Pensilvania(Ma. Auxiliadora) \\
\hline \multirow{29}{*}{$\stackrel{0}{\frac{0}{2}}$} & \multirow[t]{7}{*}{ Amambay } & \multirow[t]{3}{*}{ Capitán Bado } & Mbae Marangatu, zona 1 y 2 \\
\hline & & & Com. Ind. Piraymi \\
\hline & & & Com. Ind. Colonia Menta (Pai Tavyreta) \\
\hline & & \multirow[t]{2}{*}{ Pedro Juan Caballero } & Comunidad indígena Pirikua \\
\hline & & & Cerro Pau \\
\hline & & \multirow[t]{2}{*}{ Bella Vista Norte } & Colonia Indígena Yvy Oka \\
\hline & & & Colonia Indígena Arroyo Ka'a \\
\hline & \multirow[t]{9}{*}{ San Pedro } & \multirow[t]{3}{*}{ Tacuati } & Com. Ind. Capiatindy \\
\hline & & & Com. Ind. Jerokyroka \\
\hline & & & Com. Ind. Indígena Yvamindy \\
\hline & & \multirow[t]{4}{*}{ Santa Rosa del Aguaray } & Estancia La Yeya \\
\hline & & & Escuela agrícola de Santa Rosa del Agu \\
\hline & & & Laguna Blanca \\
\hline & & & Yaguarte Forest \\
\hline & & \multirow[t]{2}{*}{ Choré } & Nuclear dos \\
\hline & & & Comunidad 8 de diciembre \\
\hline & \multirow[t]{11}{*}{ Concepción } & Loreto & Zona Rural de Jugua Poi \\
\hline & & \multirow[t]{3}{*}{ Horqueta } & Alfonzo cue \\
\hline & & & Brasil cue \\
\hline & & & Barrio Fátima \\
\hline & & \multirow[t]{4}{*}{ Yby Yaú } & Naranjaty \\
\hline & & & Zona rural Pasiño \\
\hline & & & Cerro Memby \\
\hline & & & Est. Ganad. Cerro Cora \\
\hline & & San Lázaro & Zona de Tres Cerro \\
\hline & & Sargento Félix López & Parque Paso Bravo \\
\hline & & San Carlos del Apa & San Carlos del Apa \\
\hline & \multirow[t]{2}{*}{ Canindeyú } & Villa Ygatimi & Com. Ind. Ybypyta, zona Ñandurokai \\
\hline & & Yby Pytá & Reserva del Mbaracayú \\
\hline \multirow{8}{*}{ 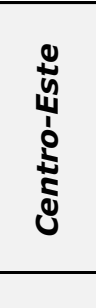 } & \multirow[t]{5}{*}{ Caazapá } & Moises S. Bertoni & Moises S. Bertoni \\
\hline & & San Juan Nepomuceno & Parque Nacional Caazapá \\
\hline & & & Cordillera del Ybyturusu \\
\hline & & Tavai & Núcleo 1 \\
\hline & & & Com. Indígena Tuna-Arroyo moroti \\
\hline & Guaira & Colonia Independencia & Compañía Pañetey \\
\hline & Alto Paraná & San Alberto & Plantaciones y bosque \\
\hline & & Hernandarias & Inmediaciones Paraná Country \\
\hline & Paraguarí & San Roque González de & Compañía Cerrito \\
\hline & & Paraguarí & Ruta $N^{\circ} 1$ Estancia Edelira \\
\hline जे & & Ybycui & Parque Nacional La Rosada \\
\hline & & La Colmena & Compañía Mbokajaty (Zona Cerro) \\
\hline & Misiones & San Ignacio Misiones & Compañía Arroyo verde, Zona rural \\
\hline & Central & Ypacaraí & Km 37 camino a Aregua \\
\hline ¿ & & & Eucaliptal desvío a San Bernardino \\
\hline$\div$ & & & km 45 Pedrozo \\
\hline & & Limpio & Compañía Salado'i (Recicladora particu \\
\hline & Capital & Asunción & Parque Guasu \\
\hline
\end{tabular}

Fuente: Elaborado por Centro Nacional de Información Epidemiológicas a partir del monitoreo de incendios forestales, agosto-octubre, 2019. 
Los departamentos con localidades afectados incluyeron a los 5 ejes geográficos, donde la mayor proporción se concentró en Alto Paraguay y en el eje norte con la totalidad de departamentos (4/4) donde se identificaron 14 distritos, de los cuales 6 eran de Concepción. Se identificaron 56 lugares afectados de los cuales 22 eran pastizales, 12 comunidades indígenas, 10 establecimientos agroganaderos, 10 parques nacionales, un cerro y la afectación de una recicladora privada en el departamento Central (Figura 1).

Fuente: Elaboración propia a partir de resumen de monitoreo de incendios forestales. Centro Nacional de Información Epidemiológica-DGVS.

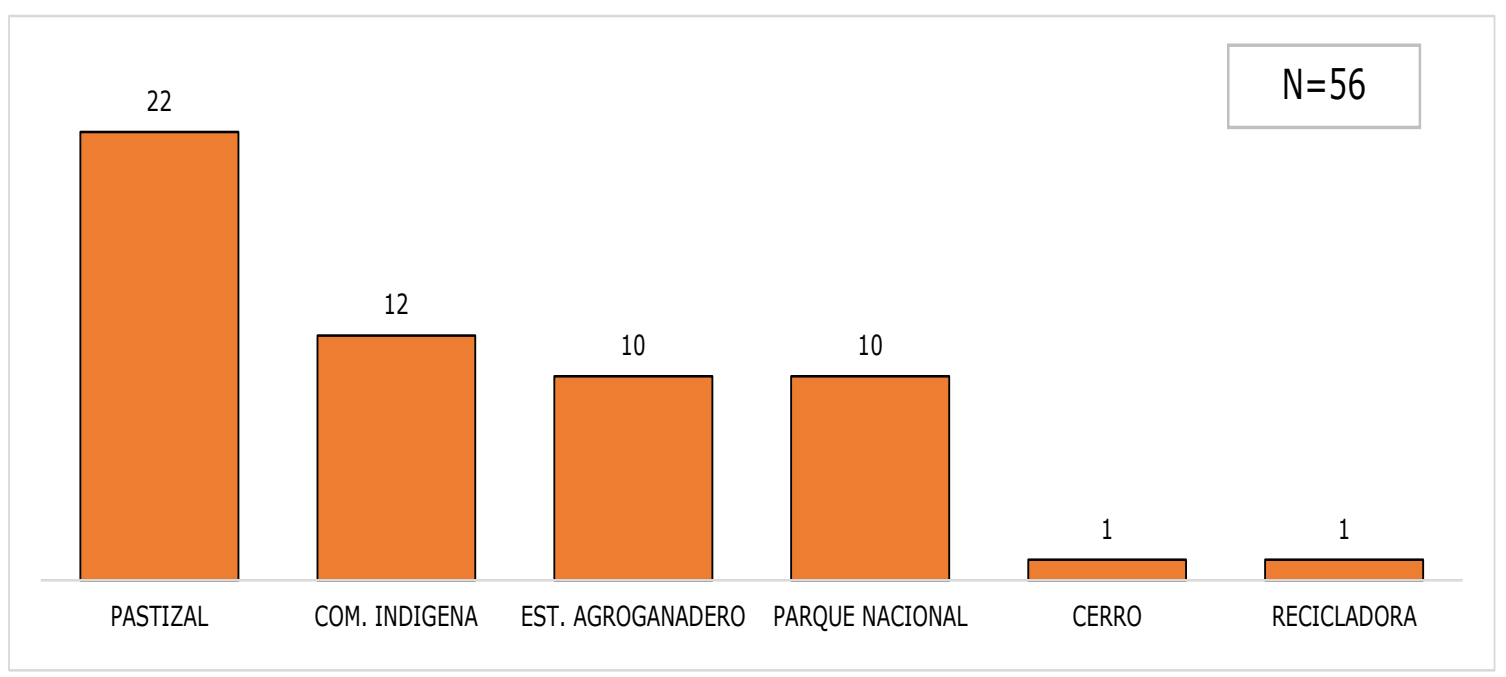

Figura 1. Tipo de lugar afectado por incendios forestales en Paraguay, del 15/08/2019 al 04/10/2019.

\section{Tipos de afectación}

La afectación incluyó pérdidas de recursos forestales, pérdida de animales silvestres en los parques nacionales y animales domésticos en estancias y comunidades indígenas.

En Alto Paraguay en la zona de Bahía Negra, el personal de las estancias y animales vacunos tuvieron que ser evacuados ante el avance de las llamas en las zonas de reservas naturales. También se sumó la falta de agua para consumo humano por la intensa sequía, principalmente en Fuerte Olimpo y la localidad de Toro Pampa, y la afectación por intensas humaredas producidas por incendios forestales en la zona del pantanal del lado brasilero, frente a Fuerte Olimpo (ambos separados por el río Paraguay).

Se reportaron pérdidas materiales en estancias y comunidades indígenas. Pérdidas materiales en $6(6 / 10)$ establecimientos agroganaderos de Alto Paraguay ${ }^{(5)}$ y Concepción ${ }^{(1)}$. En $5(5 / 12)$ comunidades indígenas afectadas, se reportaron 68 viviendas quemadas de los distritos de Tacuati, Bella Vista norte y Tavai ${ }^{(1)}$. En la comunidad indígena Capiatindy de Tacuati-San Pedro, también perdieron parte de la tubería que proveía agua, lo que ocasiono la interrupción de la provisión de agua por varios días.

\section{Tipos de enfermedades}

Se realizaron 1.681 atenciones médicas durante el evento, de los cuales 1.291 (77\%) corresponden a Alto Paraguay, 194 (12\%), Amambay, 146 (9\%) San Pedro y 50 (3\%) a Caazapá. Las enfermedades más frecuentes fueron las Infecciones Respiratorias Agudas no neumonías (36\%), las Enfermedades Tipo Influenza (18\%), la hipertensión arterial $(11 \%)$ y las diarreas $(10 \%)$. En menor proporción se registraron las irritaciones oculares y las lesiones en piel. No se registraron heridos graves, desaparecidos ni fallecidos (Figura 2). 
Fuente: Centro Nacional de Información Epidemiológica-DGVS.

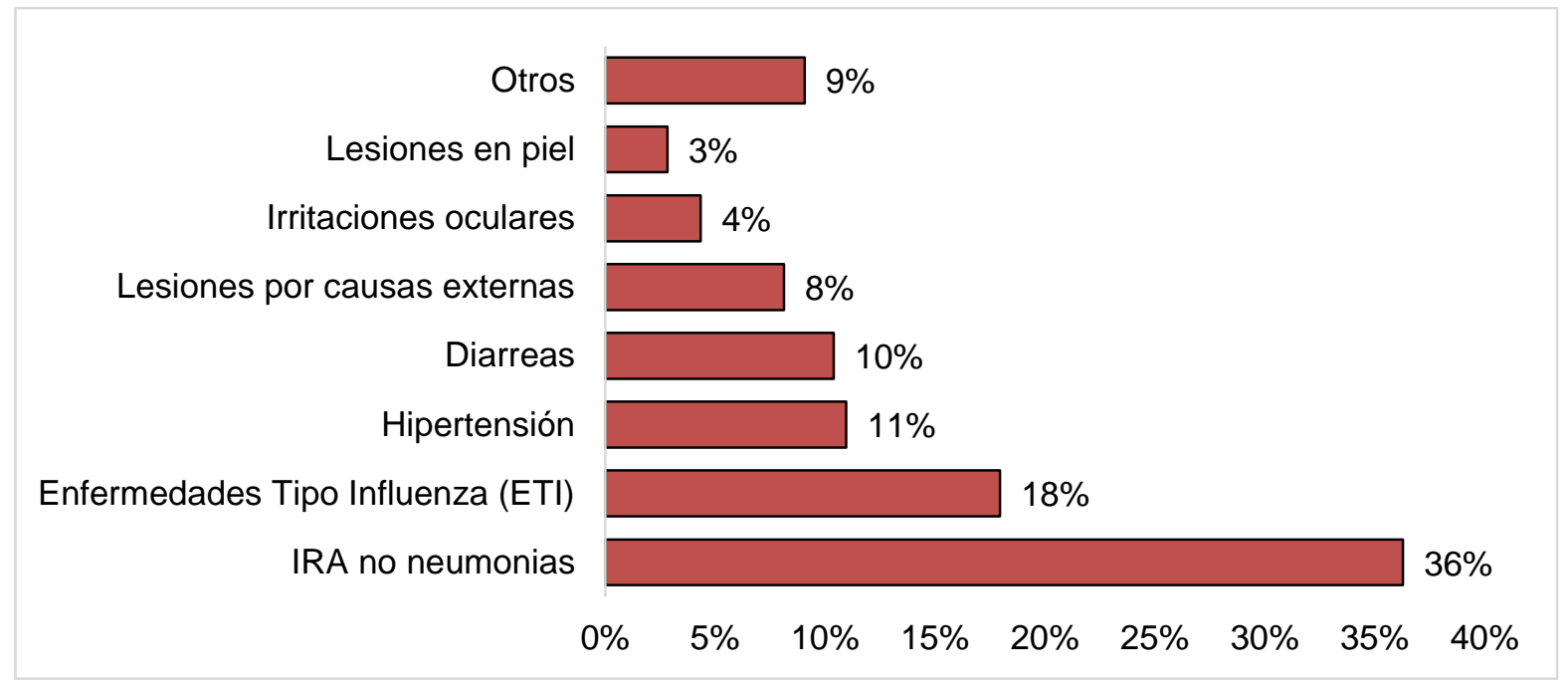

Figura 2. Proporción de enfermedades por incendios forestales en Paraguay, del $15 / 08 / 2019$ al 04/10/2019.

\section{Respuestas ante los incendios forestales Equipo multidisplinario}

- Se conformó un equipo multidisciplinario liderado por la Secretaria de Emergencia Nacional (SEN), que desplegó a sus especialistas en gestión de riesgos ambientales en las zonas críticas, en coordinación con compañías de bomberos voluntarios, personal militar, organizaciones de la sociedad civil y la Asociación Agropecuaria de Agua Dulce ${ }^{(9)}$. Además del apoyo del Ministerio de Salud Pública y Bienestar Social (MSPyBS) con sus equipos de salud.

- Para el control de incendios se implementaron acciones por aire, a través de las operaciones de 10 aviones hidrantes, puestos a disposición por los gobiernos de Chile, Brasil y Argentina, y 2 helicópteros. Asimismo, para las acciones desarrolladas en tierra, se contó con la movilización de unos 200 especialistas y pobladores locales ${ }^{(9)}$.

- La SEN en coordinación con el Instituto Forestal Nacional, el Ministerio de Medio Ambiente y Desarrollo Sostenible, la Agencia Espacial Paraguaya, la Universidad Nacional de Asunción y Fondo Mundial para la Naturaleza (WWF), informaron con reportes diarios el monitoreo de focos de incendio con mapas de posibles riesgos y pronósticos meteorológicos para el nivel nacional.

\section{Componente salud}

- Desde las Redes Integradas de Servicios de Salud (RISS) del MSPyBS, con sus hospitales, centros de salud y Unidades de Salud Familiar, realizaron atenciones médicas a la población afectada.

- Se estableció una sala de situación en el distrito de Bahía Negra, Alto Paraguay, para monitoreo del evento y atención a las personas con referentes de MSPyBS.

- Se activó la vigilancia intensificada en los departamentos afectados.

- Se emitieron recomendaciones para protegerse del humo de incendios a la población en general y se emitió la Alerta Epidemiológica $N^{\circ} 6 / 2019$, ante posibles efectos a corto, mediano y largo plazo por los incendios forestales.

- Se emitieron 10 reportes del Monitoreo en Incendios Forestales que fueron publicados en la página oficial de la Dirección General de Vigilancia de la salud (http://dgvs.mspbs.gov.py/page/\#vista eventos especiales.html) 


\section{DISCUSIONES Y CONCLUSIONES}

En el 2019, se registraron incendios forestales en más de dos tercios de los departamentos del Paraguay, con mayor afectación a los departamentos de Alto Paraguay y a los del eje norte. Se registraron daños severos en las reservas naturales más extensas del país, pérdidas materiales en establecimientos agroganadero, así como perdidas de viviendas de comunidades indígenas, se identificaron situaciones de riesgo como exposición intensa al humo de los incendios, falta de agua para consumo, así como la evacuación de comunidades. En cuanto a la salud de las personas, se registró mayor proporción de cuadros respiratorios como las IRAS no Neumonias y las ETI, seguidos por hipertensión y diarreas, no se registraron fallecidos ni desaparecidos.

Cabe destacar que, los incendios forestales del 2019 se dieron en varias regiones de Sudamérica, entre los que se mencionan los incendios en la Amazonia brasileña que afecto una extensa proporción de bosques, se estima que afecto el $55 \%$ del área despejada equivalente a más $5500 \mathrm{Km}^{2}$ ( $555.000 \mathrm{ha}$ ), zona del pantanal brasileño, bosques secos en los departamentos de Santa Cruz y Beni de Bolivia, la Reserva de la Biosfera de Calakmul (3087 ha) al sur de México. Al igual que en otros países, una de las causas de estos incendios fueron las prácticas de la preparación de terrenos para la agricultura ${ }^{(13,14)}$.

Los departamentos afectados por los incendios forestales del 2019 en Paraguay, tuvo el mismo patrón de afectación que años anteriores. Entre el 2007 y el 2017, unos 16 departamentos registraron incendios forestales, siendo los afectados más afectados San Pedro y Concepción ${ }^{(9)}$. Los incendios tuvieron lugar en los meses de agosto y septiembre, con un total acumulado de 57.183 afectados, 8 fallecidos, y 164 viviendas destruidas ${ }^{(8)}$. En los últimos 10 años, entre los meses de julio a octubre se han registrado la mayor cantidad de focos de calor en Paraguay. En julio del 2019, se registraron 9.569 focos de calor, intensificándose en los meses agosto, setiembre y octubre con 25.299, 33.820, y 22.754 focos respectivamente, en todo el territorio paraguayo $^{(15)}$. La diferencia con los años anteriores es que no se registró fallecidos ni personas heridas graves.

En cuanto a la afectación de las reservas naturales, cerca de 325.000 ha fueron afectadas por los incendios forestales en el Chaco paraguayo, aproximadamente 230.200 ha en el Monumento Natural Cerro Chovoreca y 94.600 ha en el Parque Nacional Río Negro (Pantanal). Un informe realizado por Guyra Paraguay, refiere que los índices que se observaron durante al menos todo el mes de agosto indicaron un nivel peligroso de CO de entre 500 a 1.000 ppm sobre los poblados vecinos de Bahía Negra y Puerto Diana, e incluso otros más lejanos ${ }^{(10)}$. En este contexto, también se ha vistos en otros estudios donde el humo de los incendios forestales afecta tanto a las poblaciones cercanas a los incendios forestales, pero también a las poblaciones distantes debido al transporte de contaminantes del aire a gran distancia ${ }^{(16)}$.

En cuanto a la mayor proporción de cuadros respiratorios encontrado en este estudio, es similar a lo encontrado en la literatura, donde se refiere que a enfermedad respiratoria como la condición de salud con más frecuencia. Más del $90 \%$ de estudios de metaanálisis encontraron que el humo de los incendios forestales se asoció con un mayor riesgo de enfermedades respiratorias y cardiovasculares ${ }^{(17)}$. Las poblaciones con mayor riesgo incluyen personas con enfermedad pulmonar crónica, personas mayores, niños, mujeres embarazadas y fetos ${ }^{(17,4)}$.

Para los años 2013-15 y 2017-18, la mortalidad prematura anual atribuible a incendios forestales-PM 2.5 se estimó en 54 a 240 muertes por exposición a corto plazo y 570 a 2500 muertes por exposición a largo plazo, así como muchos resultados de morbilidad cardiorrespiratoria ${ }^{(16)}$. Si bien en este estudio, no se registraron fallecidos a corto plazo, se desconoce el impacto que pudiera producir a largo plazo en las personas vulnerables.

Existen suficientes evidencias de que los incendios a gran escala de paisaje son fenómenos cíclicos recurrentes, en periodos de 10 años aproximadamente, con repeticiones intermedias generalmente a los 1-2 años de sucedido un gran evento. Estos incendios, al menos en el caso observado en 2019, aparentemente tienen un origen humano que detona un fenómeno natural de combustión espontánea, o 
progresiva, de una cantidad notable de material orgánico acumulado luego de periodos húmedos ${ }^{(9)}$.

Debido al fenómeno cíclico de incendios a gran escala, los impactos en la salud pública del humo de incendios forestales son cada más relevantes y merecen la atención de todos los que tienen la responsabilidad de las decisiones de gestión de la calidad de la tierra y el aire y la política de incendios forestales, que deben proteger la salud del público y las poblaciones en riesgo, así como las partes interesadas que se ven afectadas por la política de incendios forestales ${ }^{(6)}$.

Como limitación de este estudio, se menciona que no se dispone de datos de salud de los bomberos forestales, ni estadísticas de calidad del aire del periodo, tampoco se encontraron datos de las pérdidas forestales del resto del país. Se necesita una mejor comunicación de información procesable por parte de los funcionarios de salud pública y los profesionales de la salud para mejorar de manera más efectiva la respuesta del público, en particular aquellos que están en mayor riesgo ${ }^{(4)}$.

En conclusión, los incendios forestales del 2019, ocasionaron cuadros respiratorios leves en poblaciones afectadas por el humo de los incendios, no se registraron heridos graves, personas desaparecidas ni fallecidas. Se identificaron riesgos potenciales para la salud por la afectación de medios de vida y sustento, sobre todo en poblaciones vulnerables como las poblaciones indígenas. Se propone mejorar la información de eventos ambientales, además de desarrollar investigaciones que permitan evaluar el efecto a largo plazo de la exposición al humo producido por los incendios forestales en la salud de la población en general.

Conflictos de interes: Los autores declaran no tener conflictos de intereses.

Financiamiento: Estudio desarrollado dentro del contexto de la Vigilancia ambiental por el Centro Nacional de Información Epidemiológica dependiente de la Dirección General de Vigilancia de la Salud, Ministerio de Salud Pública y Bienestar SocialParaguay.

Contribución de autores: Sandra Irala, Mónica Arellano, participaron en la concepción y diseño de estudio e interpretación de datos, revisando el artículo y aprobando la versión final a presentar.

Fátima Vázquez, Katia Peralta, participaron en el análisis e interpretación de datos, revisando el artículo y aprobando la versión final a presentar.

María Esther Pedrozo, participo en el diseño, interpretación de datos, redacción del manuscrito, ajustes y revisión para la versión final a presentar.

\section{REFERENCIAS BIBLIOGRÁFICAS}

1. Nasi R, Dennis R, Meijaard E, Applegate $G$, Moore P. Los incendios forestales y la diversidad biológica [Internet]. Disponible en:

http://www.fao.org/3/y3582s/y3582s08 htm

2. Sandoval D. Bárbara, Reyes R. Tatiana, Oyarzún G. Manuel. Mecanismos de los efectos nocivos para la salud de la contaminación atmosférica proveniente de incendios forestales. Rev. chil. enferm. respir. [Internet]. 2019 Mar [citado 2020 Feb 11]; 35(1): 49-57. Disponible en:

https://scielo.conicyt.cl/scielo.php?scrip $\mathrm{t}=\mathrm{sci}$ arttext\&pid=S0717-

73482019000100049

3. Urbanski SP, Hao WM, Baker S. Chapter 4 Chemical Composition of Wildland Fire Emissions. Developments in Environmental Science, 2008; 8: 79-
107. ISSN: 1474-8177. Doi: 10.1016/S1474-8177(08)00004-1

4. Cascio WE. Wildland fire smoke and human health. Sci Total Environ. 2018 May 15; 624: 586-595. doi: 10.1016/j.scitotenv.2017.12.086 Epub 2017 Dec 27. PMID: 29272827; PMCID: PMC6697173.

5. Sandoval B, Reyes $T$, Oyarzún $M$. Mecanismos de los efectos nocivos para la salud de la contaminación atmosférica proveniente de incendios forestales. Rev. chil. enferm. respir. [Internet]. 2019 Mar [citado 2021 Jul 27]; 35(1): 49-57. Disponible en: http://www.scielo.cl/scielo.php?script=s ci arttext\&pid $=$ S071773482019000100049\&lng=es. http://dx.doi.org/10.4067/S071773482019000100049 
6. Chen $\mathrm{H}$, Samet JM, Bromberg PA, Tong $H$. Cardiovascular health impacts of wildfire smoke exposure. Part Fibre Toxicol. 2021 Jan 7; 18 (1): 2. doi: PMID: 33413506 ; PMCID: PMC7791832.

7. Johnston $F$, Henderson $S$, Chen $Y$, Randerson J, Marlier M, DeFries R, et al. Estimated Global Mortality Attributable to Smoke from Landscape Fires. Environ Health Perspect. 2012 May; 120(5): 695-701. doi: 10.1289/ehp.1104422.

8. Secretaría de Emergencia Nacional. Paraguay. Plan Nacional de Implementación del Marco de Sendai 2018-2022.

9. Sistema de Inventario de Efectos de Desastres. DESINVENTAR [Internet]. Paraguay. Incendios forestales 2007$2017 . \quad$ Disponible en: https://online.desinventar.org/desinven tar/\#PRY-20150828143648. Consultado 26/09/2019

10. Guyra Paraguay. Informe de Incendios en el área del Pantanal Paraguayo 2019. Asunción-Paraguay, 2019. Pag.132 [Internet]. Disponible en: http://guyra.org.py/wpcontent/uploads/2019/11/Informe-deIncendios-en-el-Pantanal-Paraguayo2019 .pdf

11. Ministerio de Salud Pública y Bienestar Social, Dirección General de Vigilancia de la Salud. Guía Nacional de Vigilancia y Control de Enfermedades Sistema Nacional de Vigilancia Epidemiológica Paraguay, 2015 [Internet]. Disponible en:

http://dgvs.mspbs.gov.py/files/guiaNaci onal/Guia-Vigilancia-2015.pdf

12. Ejecutivo promulga ley de emergencia ambiental en el Chaco. Ultima Hora. 10 de septiembre de 2019 [Internet].
Disponible

en:

https://www.ultimahora.com/ejecutivopromulga-ley-emergencia-ambiental-elchaco-n2843004.html

13. Gómez Durán, T. 2019: el año del fuego en Latinoamérica. Mongabay. 16 de diciembre 2019. [Internet]. Disponible en:

https://es.mongabay.com/2019/12/201 9-el-ano-del-fuego/

14. Human Rights Watch (HRW). "The Air is Unbearable". Health Impacts of Deforestation-Related Fires in the Brazilian Amazon. August 26, 2020 Disponible en: https://www.hrw.org/report/2020/08/2 6/air-unbearable/health-impactsdeforestation-related-fires-brazilianamazon

15. Secretaria de Emergencia Nacional, Instituto Forestal Nacional. Reporte de focos de calor sobre la República del Paraguay. [Internet]. Disponible en: https://www.wwf.org.py/?352996/Repo rtes-de-focos-de-calor-sobre-laRepublica-del-Paraguay

16. Matz CJ, Egyed M, Xi G, Racine J, Pavlovic R, Rittmaster R, Henderson SB, Stieb DM. Health impact analysis of PM2.5 from wildfire smoke in Canada (2013-2015, 2017-2018). Sci Total Environ. 2020 Jul 10;725:138506. doi: 10.1016/j.scitotenv.2020.138506 Epub 2020 Apr 6. PMID: 32302851.

17. Liu JC, Pereira G, Uhl SA, Bravo MA, Bell ML. A systematic review of the physical health impact from nonoccupational exposure to wildfire smoke. Environ Res 2015, 136: 120-32. Disponible en:

https://doi.org/10.1016/j.envres.2014. 10.015 